\title{
An Imaged Based Method for Universal Performance Evaluation of Electrical Impedance Tomography Systems
}

\author{
Yu Wu, Member, IEEE, Dai Jiang, Senior Member, IEEE, Rebecca Yerworth, and Andreas \\ Demosthenous, Fellow, IEEE
}

\begin{abstract}
This paper describes a simple and reproducible method for universal evaluation of the performance of electrical impedance tomography (EIT) systems using reconstructed images. To address the issues where common electrical parameters are not directly related to the quality of EIT images, based on objective full reference (FR) image quality assessment, the method provides a visually distinguishable hot colormap and two new FR metrics, the global and the more specific 'region of interest'. A passive 16 electrode EIT system using an application specific integrated circuit front-end was used to evaluate the proposed method. The measured results show, both visually and in terms of the proposed FR metrics, the impact on recorded EIT images with different design parameters and non-idealities. The paper also compares the image results of a passive electrode system with a matched 'single variable' active electrode system and demonstrates the merit of an active electrode system for noise interference. A figure of merit based on the FR metrics is proposed.
\end{abstract}

Index Terms - Electrical impedance tomography (EIT), EIT system performance evaluation, figure of merit, full reference method, hot colormap, image quality assessment, passive versus active electrode EIT systems.

\section{INTRODUCTION}

$\mathrm{E}$ LECTRICAL impedance tomography (EIT) is an impedance measurement technique that uses the tomography principle to reconstruct an image which illustrates the inner impedance distribution of the subject under test (SUT). This technique has been widely used in many biomedical applications, including stroke [1], cancer detection [2] and lung function monitoring [3], [4].

Given the potential of EIT, a large number of EIT systems have been developed. At the time of writing, from 2015 to 2021, a search of 'electrical impedance tomography system, and EIT system' resulted in 1039 papers in IEEEXplore alone. However, there are only a handful of papers that describe an evaluation method for EIT systems [5]-[7], and a universally adopted EIT figure of merit (FoM) is far from being recognized.

Manuscript received March 31, 2021; revised June 16, 2021. This work was supported in part by the Engineering and Physical Sciences Research Council the Engineering and Physical Sciences Research Council under grant $\mathrm{EP} / \mathrm{T} 001259 / 1$.

Y. Wu, D. Jiang and A. Demosthenous are with the Department of Electronic and Electrical Engineering, University College London, Torrington
To understand the problem, it is important to first know the operation of an EIT system which is illustrated in Fig. 1 (a). An electrical system first measures the data from the SUT, and then using reconstruction software, the data is converted into an image by solving a highly ill-posed inverse problem. Therefore, ensuring a fair evaluation and comparison of EIT system performance requires: (A) using identical SUT, (B) using identical reconstruction software, $(\mathrm{C})$ as an imaging system, the comparison should be ultimately demonstrated in terms of an image quality factor, and (D) to be widely adopted, the method should be simple and reproducible.

For point (A), the issue is that most of the EIT systems reported have been tested on their own SUT (typically a phantom using a water tank); an example is shown in Fig. 1(b) [8]-[11]. With phantom dimensions, electrode size, concentration of the saline solution and type, and the position of object inserted - all being different, comparing one system to another by virtue of image results is almost impossible.

For point (B), there exists a dominant reconstruction software used by the EIT community, namely, EIDORS (Electrical Impedance Tomography and Diffuse Optical Tomography Reconstruction Software) [12]. EIDORS is a repository of free to use software algorithms, contributed by the EIT research community, designed to run on MATLAB. In 2009 the GREIT (Graz consensus Reconstruction algorithm for EIT) was published [13]. The GREIT is a linear regularized algorithm that calculates the EIT image based on a finite element model (FEM) of the test object. The algorithm creates a difference image using, for example, two time-interleaved datasets to reflect any conductivity changes that occurred in time. The GREIT has been formally evaluated and the code is available on EIDORS.

Regarding point (C), even though electrical design comparison for EIT systems was studied in [6] and [7], a quantitatively analyzed relationship between measurement data (which could reflect the hardware performance) and image results, is yet to be established due to the nature of solving an

Place, London WC1E 7JE, U.K. (e-mail: yu.wu.09@ucl.ac.uk; d.jiang@ucl.ac.uk; a.demosthenous@ucl.ac.uk).

R. Yerworth is with the Department of Medical Physics and Bioengineering, University College London, Gower Street, London WC1E 6BT, U.K. (e-mail: r.yerworth@ucl.ac.uk). 


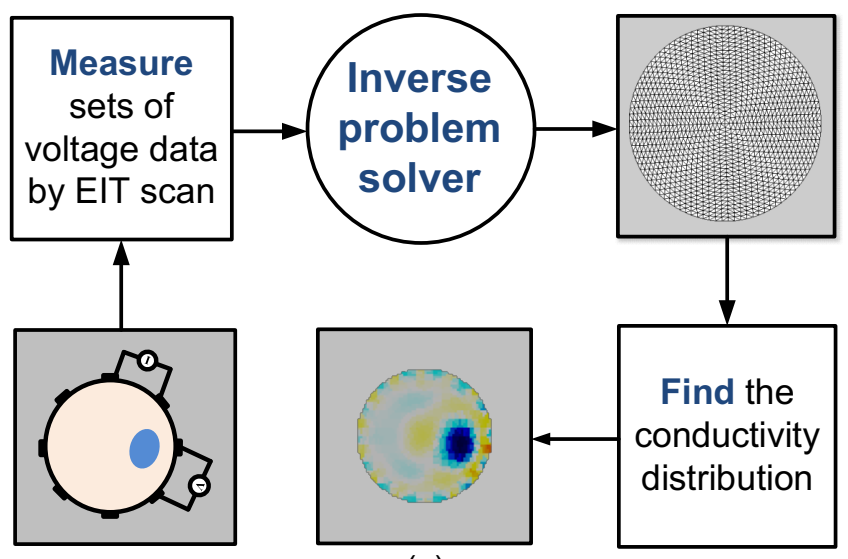

(a)

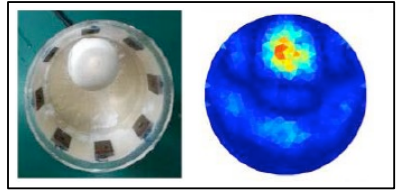

(b)

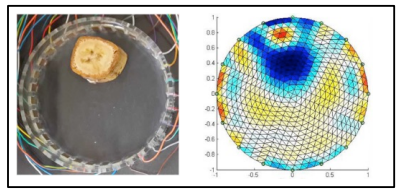

(d)

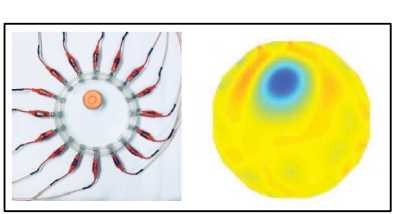

(c)

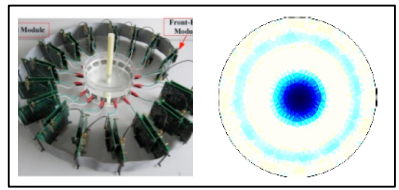

(e)
Fig. 1. (a) Overview of EIT operation. (b) $9.5 \mathrm{~mm}$ (diameter) tank with raw egg in tap-water [9], (c) $125 \mathrm{~mm}$ tank with carrot in $0.1 \% \mathrm{w} / \mathrm{v}$ salt solution [10], (d) $110 \mathrm{~mm}$ tank with banana in 'normal' saline solution [11], (e) 200 $\mathrm{mm}$ tank with nylon bar in tap water with conductivity of $347 \mu \mathrm{S} / \mathrm{cm}$ [12].

ill-posed inverse problem. Without this relationship, justification of electrical specifications or functions is difficult.

Regarding point (D), an attempt to evaluate the system performance based on image quality has been studied in [5]. However, with over ten quality parameters to define, in addition to a phantom with a robotic-arm required to reproduce the test, the approach in [5] is unlikely to be widely adopted. As EIT fundamentally does not yet offer particularly high resolution, the current practice of placing the water tank beside its EIT image [Fig. 1(b)] for visual assessment is not ideal.

In this paper, a simple and reproducible method for evaluating the performance of EIT systems is presented. The proposed full reference (FR) method is an objective image quality analysis. It computes the difference of pixels between the reconstructed image and the ground truth. Using the FR method, a passive electrode (PE) EIT system with 16 electrodes is evaluated demonstrating that the proposed FR method can establish a direct relationship between system specification and image quality. Furthermore, for the first time, a 'single variable' comparison is made between active electrode (AE) and PE EIT systems using the proposed imaged based evaluation method.

The paper builds on [14] by refining the evaluation metrics and further investigating how different design specifications can impact the image results. It also compares active and passive systems demonstrating the superiority of the former in terms of robustness against noise interference. The rest of the
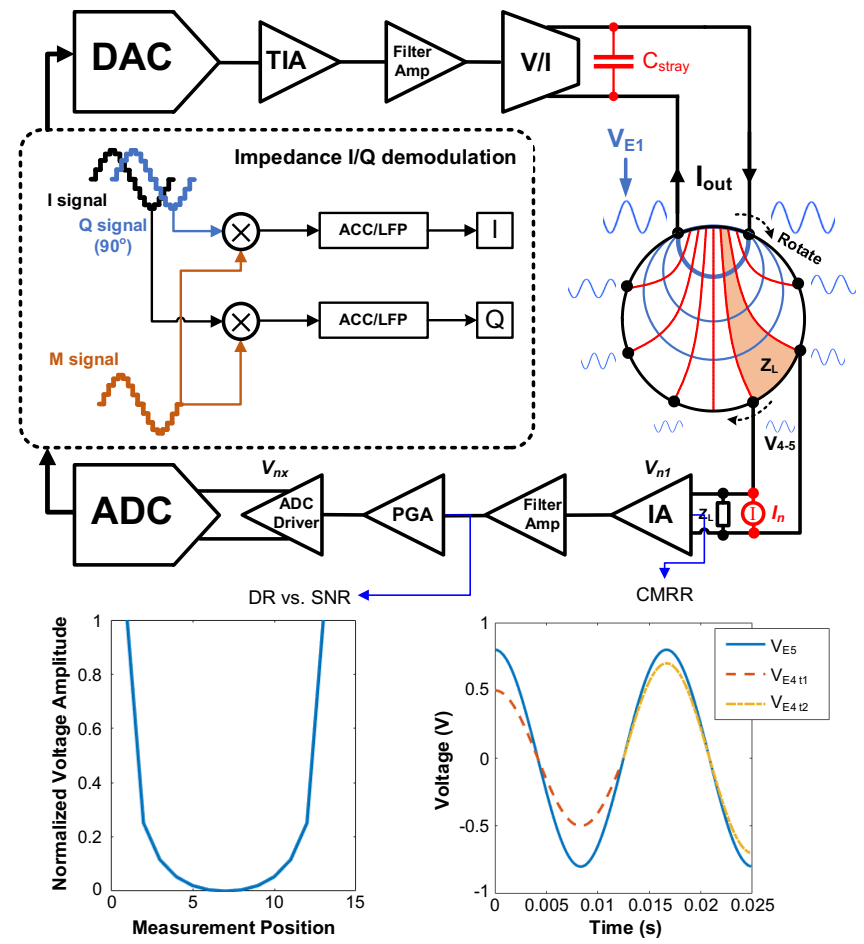

Fig. 2. EIT system architecture and impedance demodulation includes noise and non-ideality in the hardware. The I and Q signals are stored in the digital processor and the $\mathrm{M}$ signal is the measured and digitized signal from the analog recording front-end.

paper is organized as follows. Section II presents the challenges in EIT system performance evaluation. Section III presents the proposed image based assessment method and Section IV describes the results and analysis for two EIT systems using the proposed method. Concluding remarks are drawn in Section V.

\section{Challenges in EIT System Performance Evaluation}

To understand the challenges of analyzing EIT systems, a basic example of an EIT system architecture is shown in Fig. 2 to direct the analysis toward system noise sources and other non-idealities that could affect the results. It should be noted that the in-phase/quadrature-phase (I/Q) demodulation could be performed prior to analog-to-digital conversion in CMOS implementations using chopping techniques [2], [15], [16]. Various I/Q demodulation methods are reviewed in [17]. In addition, the working principle in solving the inverse problem using GREIT for image reconstruction and its challenges are also described, and finally the current popular metrics used in EIT system performance evaluation are outlined.

\section{A. EIT Measurement and Demodulation}

With an array of electrodes surrounding the SUT, the impedance measurement in EIT is performed by rotationally exciting the SUT with a small ac current. This current can be generated as shown in Fig. 2 using firstly a direct digital synthesis (DDS) to produce a voltage signal and then converting it into an output current using a voltage-to-current converter (V/I). This approach allows an adjustable output current amplitude while maintaining the full resolution of the 
digital-to-analog converter (DAC). The current is first applied to a pair of electrodes, while the voltages are recorded from the other electrodes, and this process repeats until all electrode pairs are scanned.

The voltages recorded are digitized as shown in Fig. 2 and the measured signal is used for impedance extraction. The most popular impedance extraction method is based on $\mathrm{I} / \mathrm{Q}$ demodulation and its principle is based on the relationship between the impedance $(Z)$, and the amplitude $(A)$ and phase delay $(\theta)$ of the measured voltage. This relationship can be written as $Z=R+j X=A e^{j \theta}$. The system in Fig. 2 implements I/Q multiplication and low-pass filtering in the digital domain as an example using multiply-accumulate (MAC) operation. The I and Q components can be extracted, and the impedance $Z_{L}$ and phase delay $\theta$ can be written as:

$$
\begin{gathered}
\left|Z_{L}(\omega)\right|=\sqrt{\mathrm{I}^{2}+\mathrm{Q}^{2}} \\
\theta(\omega)=\tan ^{-1} \mathrm{Q} / \mathrm{I}
\end{gathered}
$$

where $Z_{L}$ is the impedance, $\theta$ is the phase and $\omega$ is the frequency at which the impedance sample is measured. The MAC process could also be done in the analog domain where the final dc signal is digitalized by an analog-to-digital converter (ADC).

\section{B. Circuit Noise and Non-idealities}

One of the most important characteristics in any system is the signal to noise ratio (SNR) which must be evaluated. For current injection, the noise accumulates from the DAC and the V/I reflecting as a noise current $I_{n}$ in parallel with the impedance $Z_{L}$ of the SUT, which together yield an equivalent voltage noise at the input of the instrumentation amplifier (IA) as shown in Fig. 2. The additional noise in the recording chain follows the noise characteristics of cascaded amplifiers. Thus, for the $S N R_{\text {circuit }}$ for in-phase components:

$$
\begin{aligned}
& S N R_{\text {circuit }} \\
& =\frac{\left(I_{\text {out }} \cdot Z_{L} \cdot G\right)^{2} \cdot \frac{S}{2}}{\left[G \cdot\left(4 K T \cdot \operatorname{Re}\left\{Z_{L}\right\}+Z_{L} \cdot I_{n}\right)\right]^{2}+\frac{q^{2}}{12}+V_{n}{ }^{2}}
\end{aligned}
$$

where $I_{\text {out }}$ is the output current amplitude, $\operatorname{Re}\left\{Z_{L}\right\}$ is the real part of the load impedance, $G$ is the total gain of the recording chain, $S$ is the total samples accumulated in the MAC, $I_{n}$ is the equivalent noise injected by the excitation circuits, and $V_{n}$ is the total noise of the recording chain. The term $4 K T \cdot Z_{L}$ is the thermal noise of the load and $q^{2} / 12$ is the quantization noise of the ADC (The equation above is derived based on the approach in [6] and [18]). Given the large variation of ways for implementing the excitation and recording circuits, the composition of $I_{n}$ and $V_{n}$ can vary.

With reference to (3) and Fig. 2, in EIT the system SNR can vary during an EIT scan (rotation). The equivalent $Z_{L}$ is constantly changing while voltages are recorded from different electrode pairs. As shown in Fig. 2, the voltage amplitude reduces as the recording electrodes are far from the current injection site. This forms a ' $U$ ' shape pattern to be compensated by the programmable gain amplifier (PGA) and results in a different value of $G$. Thus, an accurate equivalent system SNR calculation for the EIT system can be difficult.

In addition to SNR there are other non-ideal factors in an EIT system that must be considered. Importantly the current driver must be fully differential to avoid large common mode signals [19], and any stray capacitance at the output of the current driver degrades the output impedance leading to an inaccurate output current. On the recording side, the common mode rejection ratio (CMRR) of the IA is also important [20]. As shown in Fig. 2, the IA measures the variations of differential voltages between two electrodes (in time differencing EIT). The impedance variation is reflected accurately only if the IA can reject the common mode signal at the EIT frequency. Detailed analysis of EIT systems and design considerations are reviewed in [17].

\section{The GREIT Algorithm}

GREIT belongs to a class of algorithms which use FEM to calculate the voltages that would be measured when defined current injection patterns are applied, then use matrix inversion to generate a mapping (sensitivity matrix) from the measured boundary voltages to an internal conductivity image. However, as previously stated, this is a very ill posed problem. There is not enough information in the boundary voltage measurements to independently determine the conductivities of every individual element (pixel or voxel) in the image. Instead, assumptions must be made, for example, about the smoothness of the conductivity changes. There are many different approaches to this 'regularization', with advantages and disadvantages to each approach. In the GREIT algorithm uniformity of response across the image is prioritized, but nevertheless artefacts can be present, particularly near the electrodes [13]. Another issue is the necessary assumption that there is a linear relationship between internal conductivities and the boundary voltages, but this is only valid for small changes in conductivity. Therefore, difference imaging is often used, i.e., images of conductivity change are reconstructed by multiplying the change in boundary voltage (measured boundary voltage under reference conditions) by the sensitivity matrix. The use of this difference (also known as functional imaging) approach has added advantages in that it reduces the impact of mismatches between the FEM and the SUT (e.g., boundary shape, electrode position and size, conductivity distribution of the reference condition) [21].

\section{Current EIT System Evaluation Metrics}

As a well-developed technology, many metrics have been proposed for EIT or bio-impedance system evaluation. The most popular are:

\section{1) Equivalent measurement sensitivity (resolution):}

$$
R_{\text {sense }}=\frac{V_{\text {noise }}}{I_{\text {out }}}
$$

where $V_{\text {noise }}$ is the noise of the analog front-end and $R_{\text {sense }}$ is the measurement sensitivity in $\Omega / \sqrt{\mathrm{Hz}}$ [22], [23]. The 
$R_{\text {sense }}$ value can reflect the front-end performance but does not reflect the overall EIT system performance.

2) SNR for channel variation:

$$
\begin{gathered}
S N R_{i}=\left(\frac{\sum_{n=1}^{N} V_{m}}{\sum_{n=1}^{N}\left|V_{m}-\overline{V_{m}}\right|}\right)_{i} \\
\overline{S N R_{d B}}=20 \log _{10} \frac{1}{i_{\text {max }}} \sum_{i=1}^{i_{\max }} S N R_{i}
\end{gathered}
$$

where $i_{\text {max }}$ is the total number of measurement channels in the system, e.g., for a $x$-electrode system, $i_{\text {max }}=x$. $(x-3)$ and $S N R_{i}$ is calculated for each channel. $N$ is the total number of frames measured for this SNR calculation, $V_{m}$ is the measured channel voltage and $\overline{V_{m}}$ is the average voltage measured within channel $i$ over $N$ frames. $\overline{S N R_{d B}}$ only shows the channel variation and the system measurement repeatability, and was used in [24], [25].

3) Measurement accuracy:

$$
\begin{gathered}
A C C_{i}=\left(1-\frac{\sum_{n=1}^{N}\left|V_{m}-V_{\text {ref }}\right|}{N \cdot V_{\text {ref }}}\right)_{i} \cdot 100 \% \\
\overline{A C C}=\frac{1}{i_{\max }} \sum_{i=1}^{i_{\max }} A C C_{i}
\end{gathered}
$$

where $\overline{A C C}$ is the overall percentage error, $V_{\text {ref }}$ is a reference voltage obtained through simulation and the other symbols are as previously defined in (5). It requires a reference SUT that can both be simulated and measured [24].

4) FoM by $S N R[4]$ :

$$
F O M_{S N R}=\frac{P_{\text {system }}}{f \cdot 10^{\frac{\overline{S N R_{d B}}}{20}}}
$$

where $P_{\text {system }}$ is the system power consumption and $f$ is the system operating frequency. $P_{\text {system }}$ is included because a higher current injection provides a better SNR. Also, a wider EIT bandwidth can be used but at the expense of a higher power consumption; $\overline{S N R_{d B}}$ is defined in (5).

All the above evaluation metrics provide limited information on the different aspects of the system's performance. Importantly, for an imaging system, none of the above metrics relate to image quality. Therefore, a universal FoM for evaluation of EIT systems is required which can facilitate direct comparison of image quality. It would also help to better justify the choice of electrical specifications for EIT system design and promote further EIT hardware development.

\section{Image Based Evaluation Method}

\section{A. Model Simulation for Concept Illustration}

An image based EIT FoM must assess the image quality produced by the EIT system. Since it is not possible to perform EIT image quality assessment subjectively due to its low

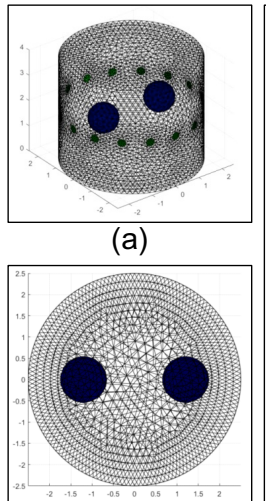

(b)

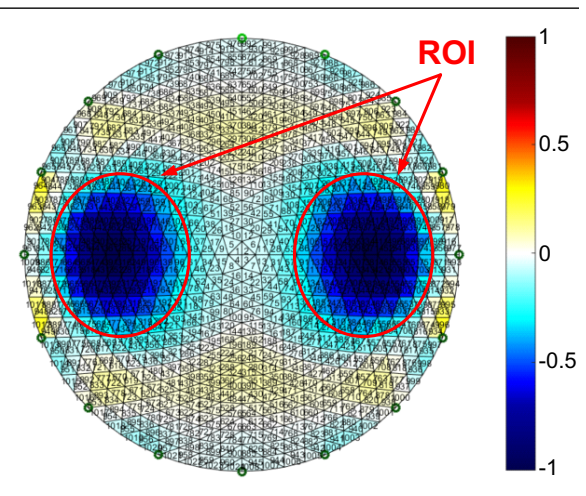

(c)
Fig. 3. (a) 3D forward model. (b) Top view of the 3D model. (c) EIT image generated using the simulation data. The color bar indicates normalized conductance and is applied throughout this paper.

resolution, an objective FR method is proposed. The proposed FR method compares the color accuracy of the test image to a reference image which is considered as the ground truth. Pixel by pixel, this FR comparison identifies the errors in the test image to determine EIT image quality.

The concept of the proposed method is illustrated by a simulation shown in Fig. 3. The software package used in the study is EIDORS v3.9.1. Firstly, the reference image (ground truth) must be generated. This is done by solving a forward problem. The forward model is built as shown in Fig. 3(a). It comprises two less conductive spheres inside a homogenous tank model. They are inserted at the center plane of the tank to cause an inhomogeneous conductive re-distribution. By placing the electrodes on the center plane of the tank and solving the forward problem, the ideal homogeneous and inhomogeneous datasets can be generated for the ground truth. To illustrate the concept, using a 1024-pixel model, Fig. 3(c) shows the reconstructed reference image corresponding to the physical model in a cross-section view as shown in Fig. 3(b). The color in the pixels uses by default an 8-bit resolution color code with the blue color representing higher resistivity region. The regions of interest (ROI) show the locations of inhomogeneous conductive re-distribution identified in Fig. 3(c).

In this simulation illustration, the test images were generated by applying a random percentage variation in the inhomogeneous dataset of the ground truth to generate $N$ frames of 'noisy/corrupted' images $(N=50)$. The proposed method calculates a FR metric for each pixel averaged over $N$ frames of images using the expression below:

$$
F R_{x}=0.5 \cdot \frac{\sum_{n=1}^{N} \mid \text { EDtest }_{x}-\text { EDref }\left._{x}\right|_{n}}{N}
$$

where $x$ represents the pixel number, EDtest $t_{x}$ and EDref $_{x}$ are the element data of each pixel stored in the inverse solved EIT models. The color code used in each pixel is mapped from the element data. The element data has 64-bit precision and is normalized to a range from -1 to 1 . With normalization, the maximum possible $F R_{x}$ metric is 2; a factor of 0.5 scales $F R_{x}$ to a range from 0 to 1 for simplicity. $F R_{x}$ can be used instead of the original pixel color code to generate a 'hot colormap' 

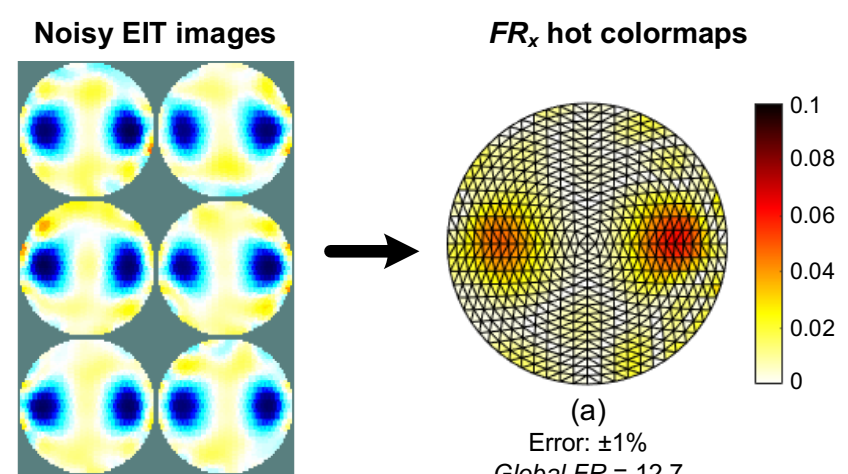

(a)

Error: $\pm 1 \%$

Global $F R=12.7$

$R O I F R=5.4$
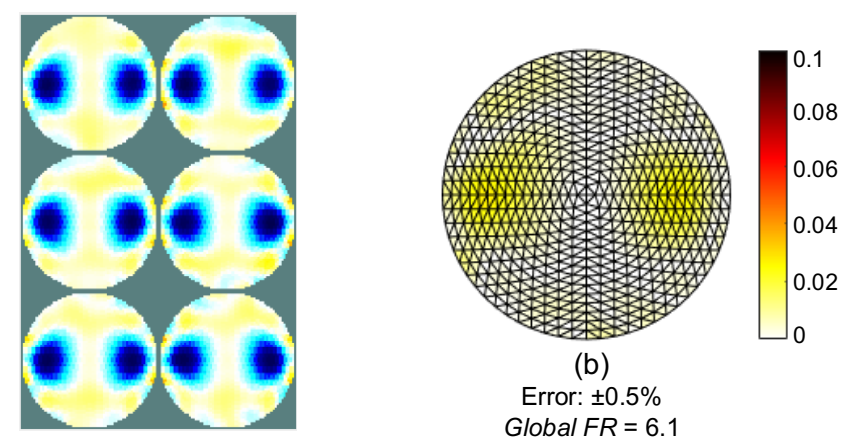

(b)

Error: $\pm 0.5 \%$

Global $F R=6.1$

$R O I F R=2.7$

Fig. 4. $F R_{x}$ hot colormaps with the original EIT images: (a) $\pm 1 \%$ variation noise added, (b) $\pm 0.5 \%$ variation noise added. $F R_{x}$ hot colormaps using 'flipud(hot)' MATLAB function. The 'ground truth' reference image is in Fig. 3(c).

image to better distinguish the errors between the test image and the reference image for better visual identification.

As shown in Fig. 4, two random percentage variations were added to the test images; $\pm 1 \%$ and $\pm 0.5 \%$. On the left of Fig. 4 are six conventional EIT images selected out of the 50 frames, and on the right is the $F R_{x}$ hot colormap over 50 frames of test images. With the $F R_{x}$ hot colormap is much easier to identify EIT image quality.

Note that the highest plotting scale (color bar) for $F R_{x}$ is limited to 0.1 for reasons of color consistency; any errors above 0.1 are considered significant and are represented by black color. To provide a quantitative comparison including all errors, even those above 0.1, two additional metrics, GlobalFR $R O I F R$, are proposed and shown in Fig. 4 where

$$
\text { Global FR }=\sum_{x=1}^{1024} F R_{x}
$$

and $F R_{x}$ is from (8). ROI $F R$ selects pixels within the ROI for summation [for ROI definition refer to Fig. 3(c)].

A summary is provided below to assist the image interpretation:

1. The original EIT image is plotted with blue/white/red contrast corresponding to higher/no/lower resistance change in the region.

2. The hot colormap is a image with each pixel in the EIT image refilled with the calculated $F R_{x}$; the pixels with larger error between reference and test image are darker.

3. Global FR and ROI FR are the total sum and selective

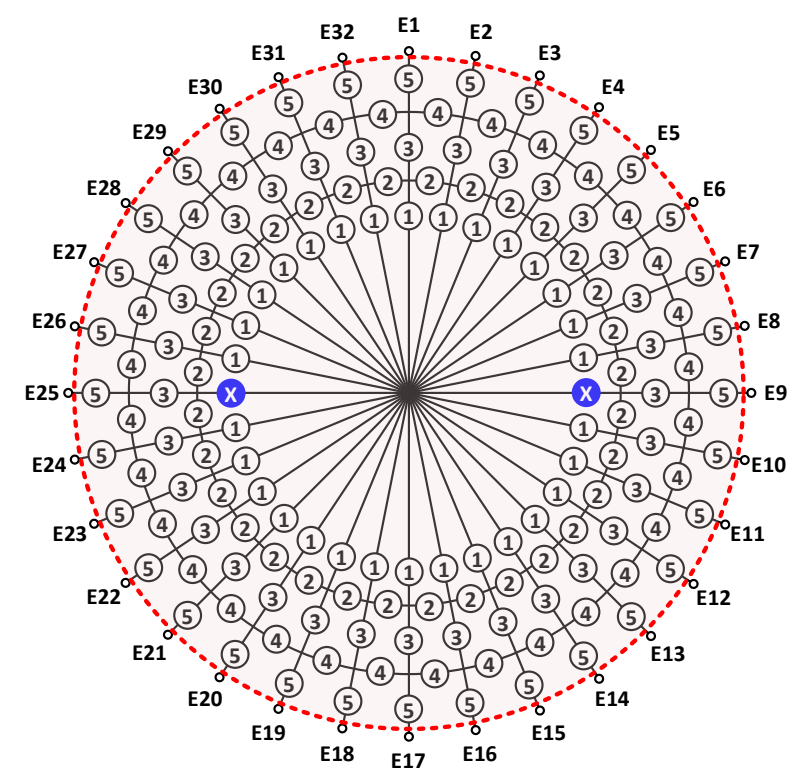

Fig. 5. EIT resistive phantom used as the ground truth for FR evaluation. Resistive elements (5) $=100 \Omega$, (4) $=196 \Omega$, (3) $=63.4 \Omega$, (2) $=6.34 \Omega$, (1) $=68.1 \Omega$. X toggled between $0 \Omega$ and $68.1 \Omega$.

sum (based on ROI) of $F R_{x}$ in the hot colormap, respectively.

4. Higher Global FR and ROI FR indicate worse images.

\section{B. Reference Image Generation}

For hardware system evaluation, a resistive phantom [26] is used instead of a water tank as the ground truth. A 32-electrode phantom (Sentec AG, Switzerland) shown in Fig. 5 is easy to implement and commercially available. By skipping electrodes, the phantom can be used for 8,16 , or 32 electrode systems. These electrode numbers are commonly used in EIT systems as shown in Fig. 1. Many recently published EIT systems use 16 electrodes [2], [4], [25], [27] so a 16 electrode system was chosen as an illustrative example, and evaluated using the proposed method.

The ideal dataset can be generated using most of the electrical simulation tools. In the resistive phantom, the two $\mathrm{X}$ resistive elements can be toggled between $68.1 \Omega$ and $0 \Omega$ for homogeneous and inhomogeneous datasets for EIT differential imaging. The dataset for the ideal reference image used in this FR comparison study was generated through simulation using Cadence Virtuoso (Cadence Design Systems, Inc., USA) with adjacent EIT scan pattern [28]. Benefiting from differential imaging, and the fact that the phantom uses purely resistive elements, for simulation data, the amplitude and frequency of the current drive has no effect on the final image result.

With respect to comparing EIT hardware performance, it is more important that all test conditions are kept constant, than that they are 'optimal'. Hence the decision to use the default GREIT 'hyper-parameters', which control the regularization within this algorithm. The following parameters are used in EIDORS: 
$\% \%$ FR-metric FEM model

n_elecs $=16$;

FEM mod $=$ ' $\mathrm{d} 2 \mathrm{c}$ ';

imdl = mk_common_model(FEM_mod,n_elecs);

imdl.fwd model.stimulation $=\mathrm{mk}$ stim patterns $(\mathrm{n}$ elecs, 1 ,

$[0,1],[0,1],\{$ 'rotate_meas', 'no_meas_current' $\}, \overline{3}$ );

The FEM is based on a 16-electrode homogeneous cylindrical object ' $\mathrm{d} 2 \mathrm{c}$ ' with circular electrodes. The ' $\mathrm{d} 2 \mathrm{c}$ ' FEM model has 1024 pixels. The 'rimgO' is the previously mentioned inverse solved EIT model where 'ref' and 'data' are the input EIT data for differential imaging.

The 'elem_data' $\left[E D_{\text {ref }}\right.$ in (8)] is the mentioned element data stored in 'rimgO' for each pixel. The 'mapped_color $=127$ ' and 'ref_level $=0$ ' set color code 127 as white color. Color code 256 is red (conductive) and 0 is blue (resistive). The ROI pixels are selected with a color code less than half of the 'mapped_color' in this study. The color code used (shown below) is the same as the default setting and does not affect the FR computation.

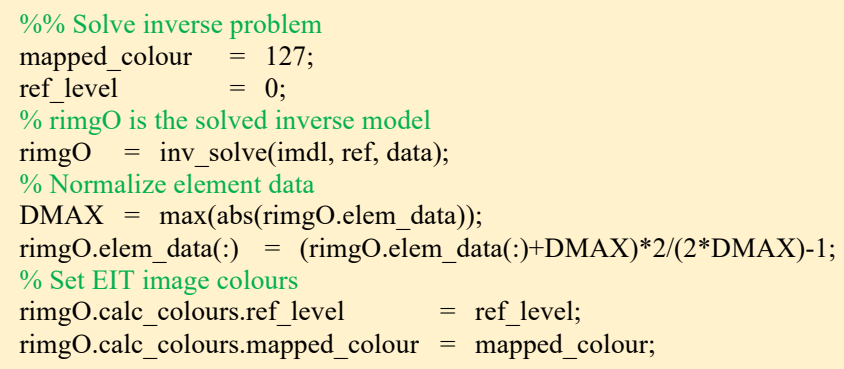

A physical phantom was designed on a PCB using $1 \%$ tolerance resistors to minimize the error between simulation and measured data. Resistor tolerance can be neglected, provided a difference in FR metrics can be demonstrated when bench-marking other controlled variables e.g., errors which are mostly contributed by the EIT system itself.

\section{Hardware Implementation}

A 16-electrode PE EIT system was implemented to evaluate the proposed FR method. In a PE EIT system, there are no active electronics at the point of contact to the SUT. The SUT is connected to the EIT system using a cable. Fig. 6(a) shows the architecture of the system. The scan cycles for current drive and voltage recording are facilitated by an analog switch matrix, which selectively connects the 16 electrodes to either the positive or negative output of the fully differential current driver (CD), or the positive or negative input of the first stage IA (IA1). The CD and IA1 are integrated in a high performance application specific integrated circuit (ASIC) in $0.35 \mu \mathrm{m}$ CMOS technology [27].

The CD can generate fully differential ac sinewave currents for frequencies up to $1 \mathrm{MHz}$ with a maximum peak-to-peak amplitude of $6 \mathrm{~mA}$ (the maximum allowed safe current amplitude is detailed in [17]). For EIT measurement, the CD is driven by a sinusoidal signal generated from a 12-bit DAC, where the input to the DAC is from the look-up table inside a Xilinx Artix-7 FPGA. The frequency and amplitude of the DAC output can be programmed to vary the drive current for EIT

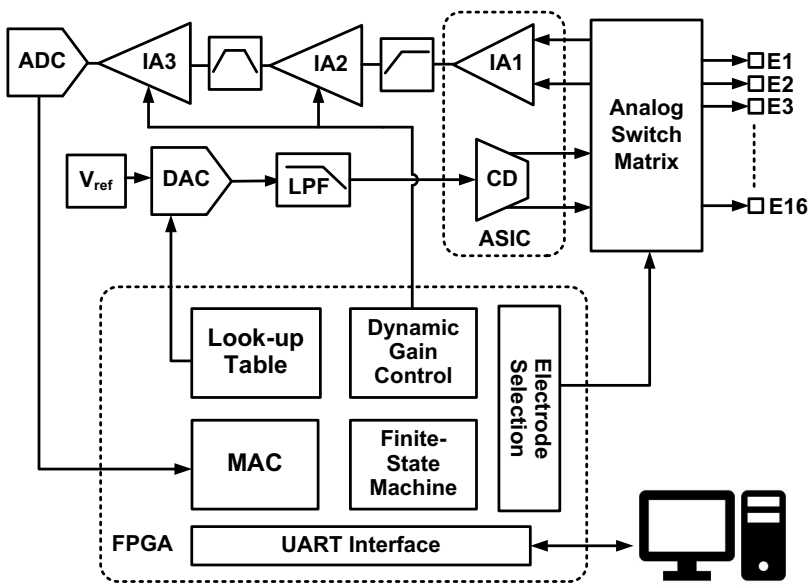

(a)

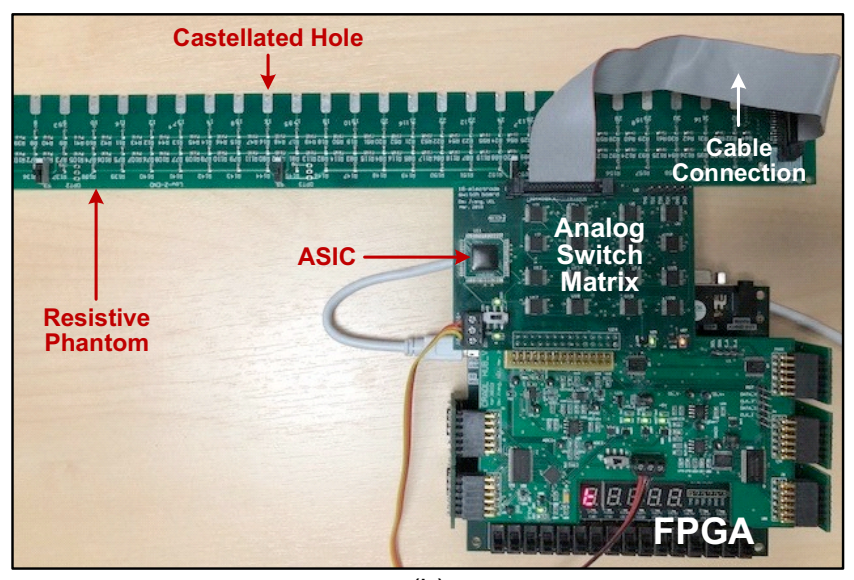

(b)

Fig. 6. (a) System architecture of a 16-electrode PE EIT system. (b) System test set-up.

measurement. The voltage on the selected electrode pairs in an EIT scan is amplified in three stages. The first stage integrated IA1 has a fixed gain of $10 \mathrm{~V} / \mathrm{V}$. The gains of the second and third stage amplifier, IA2 and IA3, are dynamically adjusted by the FPGA during an EIT scan, depending on the relative distance between the voltage recording electrode pair and the current drive electrode pair. The total gain of the 3-stage amplification is adjustable between $10 \mathrm{~V} / \mathrm{V}$ and $2,000 \mathrm{~V} / \mathrm{V}$. The amplified voltage is digitized by a 12-bit ADC, and then processed in a MAC in the FPGA to derive, using coherence detection, the real and imaginary values of the measured impedance. The results are sent to a computer via a UART interface for image reconstruction. A finite-state machine in the FPGA controls the operation of the system.

\section{System Evaluation Using FR Metrics}

To provide fair comparisons using the proposed method, the following conditions should be met:

1. The EIT system should use 'adjacent scan' for evaluation.

2. EIT systems with the different electrode counts should not be compared. 


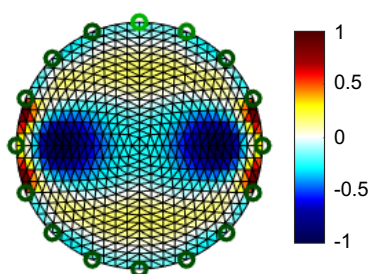

(a)

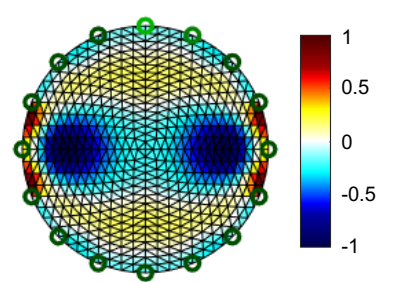

(b)

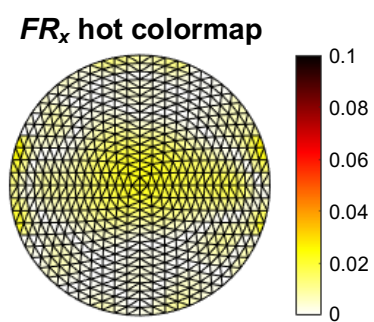

(c)

Global $F R=7.4$

$R O I F R=1.4$
Fig. 7. Reconstructed EIT image using (a) resistive phantom simulation data (reference image) and (b) PE system measured data (test image). (c) The resulting $F R_{x}$ hot colormap.

3. The EIT image contrast should be created by toggling the $\mathrm{X}$ element shown in Fig. 5 between $0 \Omega$ and 68.1 $\Omega$

4. The GREIT algorithm should be used as shown in the paper.

The PE EIT system was tested on the phantom using the adjacent EIT scan pattern. The test equipment is shown in Fig. 6(b). The drive current amplitude was set to $4 \mathrm{~mA}_{\mathrm{p}-\mathrm{p}}$ with a frequency of $125 \mathrm{kHz}$ and capturing 50 EIT data frames at a rate of 122 frames/s and with dynamic gain control. As mentioned, by selecting pixels with a value half of the 'mapped_color' locates the ROI. Using the proposed FR metrics and generating the $F R_{x}$ hot colormap, the imaging performance of the PE EIT system is shown in Fig. 7. The measured element data $\left[E D_{\text {test }}\right.$ in (8)] is normalized using $95 \%$ of the total 50 frames of data in finding the DMAX to avoid noise spikes as shown below:

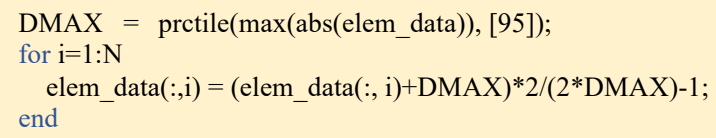

Fig. 7(a) is the reference image reconstructed using ideal data obtained from simulation of the resistive phantom shown in Fig. 5. Fig. 7(b) is the image reconstructed using measured data from the physical version of the PE EIT system of the same resistive phantom. The differences between the reference and measured images are illustrated by the $F R_{x}$ hot colormap in Fig. 7(c). The global FR metric reflects the overall image quality and the ROI represents the object's identifiability.

\section{A. Impact of Different EIT Parameters}

The impact of various design parameters was analyzed using the proposed FR method and the results are shown in Fig. 8:

\section{(1) Excitation current amplitude:}

The best FR metrics that this PE system can offer are

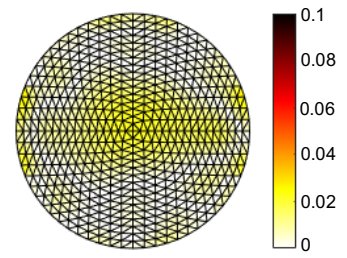

(a)

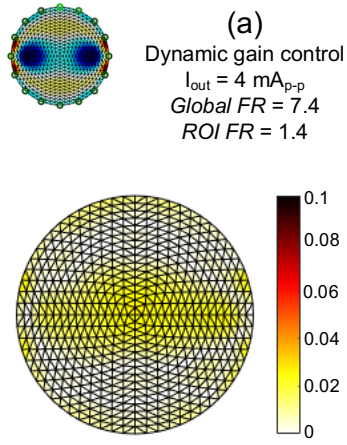

(c)

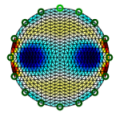

Fixed gain of $200 \mathrm{~V} / \mathrm{V}$ $\mathrm{I}_{\text {out }}=4 \mathrm{~mA}_{\mathrm{p}-\mathrm{p}}$ Global $F R=9.1$ $R O I F R=2$

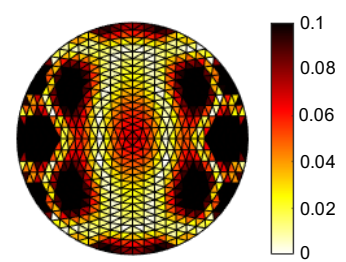

(e)

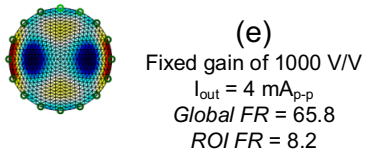
$R O I F R=8.2$

Measured image has a more conductive background

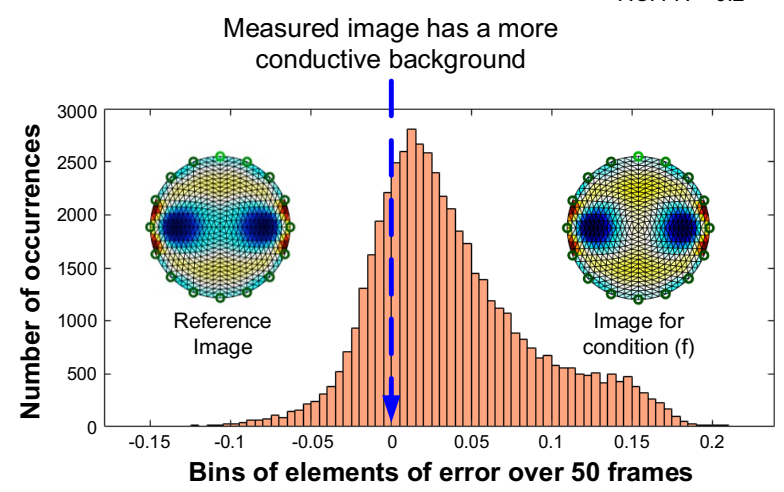

(g)

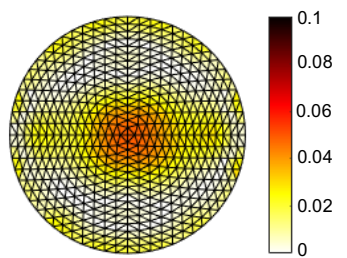

(b)

Dynamic gain control $\mathrm{I}_{\text {out }}=500 \mu \mathrm{A}_{\mathrm{p}-\mathrm{p}}$ Global $F R=14.4$
$R O I F R=2.3$

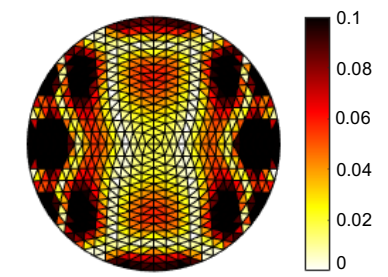

(d)

Fixed gain of $500 \mathrm{~V} / \mathrm{N}$ $\mathrm{I}_{\text {out }}=4 \mathrm{~mA}_{\mathrm{p}-\mathrm{p}}$ Global $F R=58$ $R O I F R=7.1$

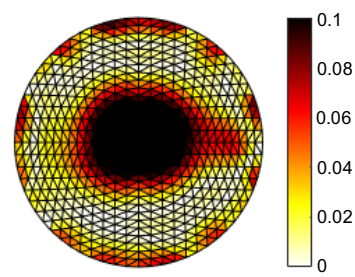

(f)

Dynamic gain contro $\mathrm{I}_{\text {out }}=4 \mathrm{~mA}_{\mathrm{p}-\mathrm{p}}$ armonics $\begin{array}{rl}R O I & F R \\ & =6.2\end{array}$

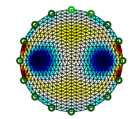

Fig. 8. PE EIT system $-F R_{x}$ hot colormaps and the reconstructed EIT images using different conditions: (a) dynamic gain and $4 \mathrm{~mA}_{\mathrm{p}-\mathrm{p}}$ current drive, (b) dynamic gain and $500 \mu \mathrm{A}_{\mathrm{p}-\mathrm{p}}$ current drive, (c) $200 \mathrm{~V} / \mathrm{V}$ fixed gain and $4 \mathrm{~mA}_{\mathrm{p}-\mathrm{p}}$ current drive, (d) $500 \mathrm{~V} / \mathrm{V}$ fixed gain and $4 \mathrm{~mA}_{\mathrm{p}-\mathrm{p}}$ current drive, (e) $1000 \mathrm{~V} / \mathrm{V}$ fixed gain and $4 \mathrm{~mA}_{\mathrm{p}-\mathrm{p}}$ current drive, and (f) dynamic gain with $4 \mathrm{~mA}_{\mathrm{p}-\mathrm{p}}$ current drive and $40 \mathrm{~dB}$ harmonic distortion. (g) Histogram of the error in element data over 50 frames for condition (f).

Global $F R=7.4$ and $R O I F R=1.4$ as shown in Fig. 7(b) and Fig. 8(a). While keeping other parameters unchanged, the impact of a lower current injection was investigated; the excitation current was reduced from $4 \mathrm{~mA}_{\mathrm{p}-\mathrm{p}}$ to $500 \mu \mathrm{A}_{\mathrm{p}-\mathrm{p}}$. 


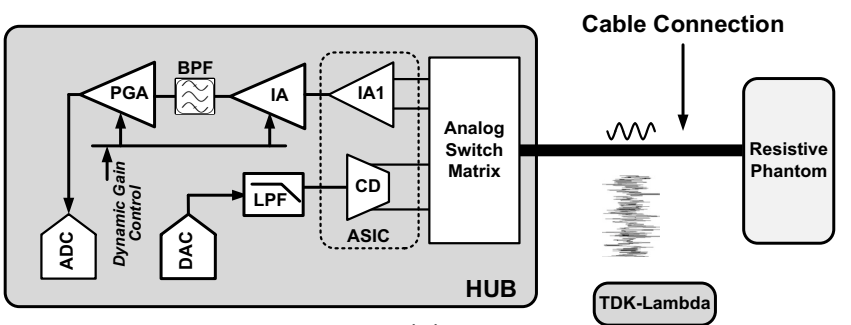

(a)

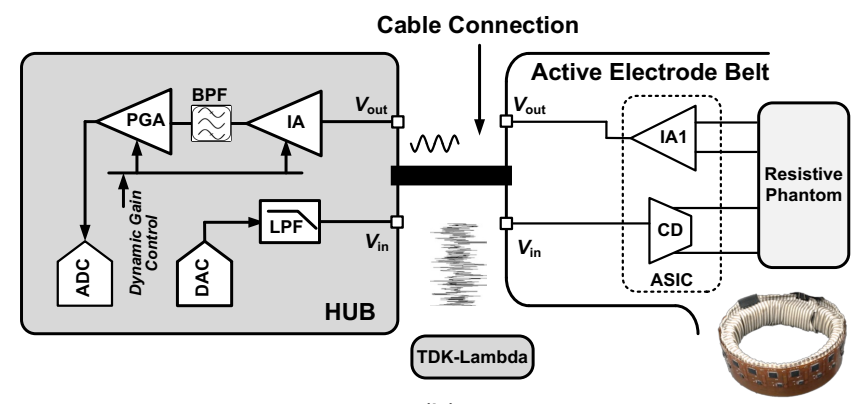

(b)

Fig. 9. (a) Developed PE EIT system in a remote setting with cable placed over the dc/dc supply (b) The same arrangement for AE EIT system.

The computed Global FR was increased to 14.4 and ROI FR to 2.3 as shown in Fig. 8(b).

\section{(2) Dynamic vs. fixed gain:}

With reference to (3), the correlation of the proposed FR metrics with the conventional circuit noise-based SNR metric was investigated. The impact on image quality was evaluated when the voltage gain in the recording channel was changed from dynamic gain to a constant gain of $200 \mathrm{~V} / \mathrm{V}$ while keeping other parameters unchanged. As shown in Fig. 8(c) Global $F R=9.1$ and $R O I F R=2$ were computed.

The proposed FR metrics can also be used to investigate the impact on the image results of other gain settings. In EIT, as a design tradeoff, some non-dynamic gain designs choose higher gains to increase the SNR of the electrode voltage recordings far from the excitation electrodes at the expense of saturating the recordings from the closely electrodes. To investigate this further, constant gains of $500 \mathrm{~V} / \mathrm{V}$ and $1000 \mathrm{~V} / \mathrm{V}$ were used, and the results are shown in Fig. 8(d) and (e), and it is evident that it is a poor tradeoff.

\section{(3) Harmonics within the excitation signal:}

While keeping other parameters unchanged, a $40 \mathrm{~dB}$ second harmonic was introduced into the DDS look-up-table. Without careful subjective comparison, the original EIT images appear to be similar compared to the reference image. However, as shown in Fig. 8(f) Global FR and ROI FR increased to 43.4 and 6.2, respectively. Plotting the errors from 50 frames in a histogram [Fig. $8(\mathrm{~g})$ ], and cross referencing to the $F R_{x}$ hot colormap in Fig. 8(f), it can be concluded that the element data [refer to (8)] in the measured image is generally shifted toward red (a conductive background) than expected, and that mostly occurs in the center of the image.
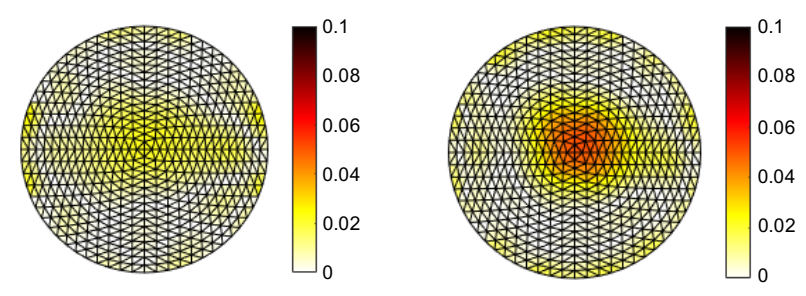

(a)

PE EIT, no interference Global $F R=7.4$ $R O I F R=1.4$

(b)

AE EIT, no interference Global $F R=10.8$ $R O I F R=1.3$
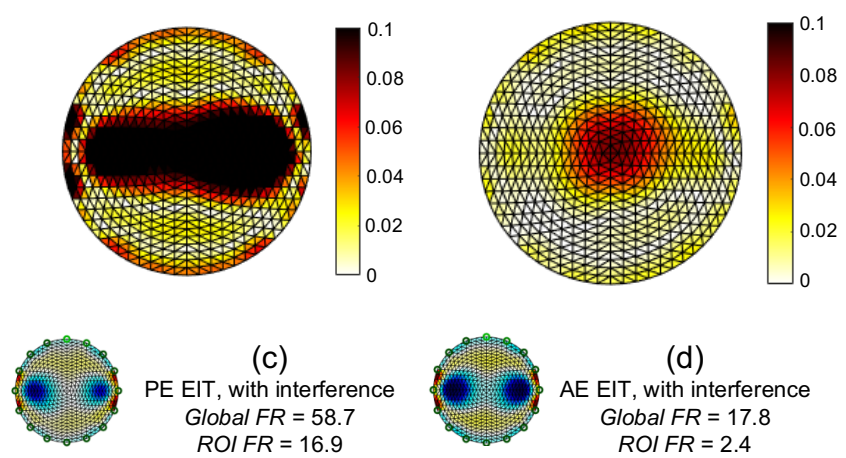

(c)

PE EIT, with interference Global $F R=58.7$ $R O I F R=16.9$

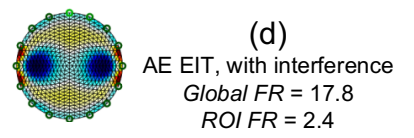

Fig. 10. $F R_{x}$ hot colormaps and the corresponding EIT images: without supply interference for (a) PE and (b) AE, and with supply interference for (c) PE and (d) AE.

\section{B. Comparison Between Active and Passive Electrode EIT}

In contrast to the PE EIT system, the AE EIT system places the analog front-end electronics on the contacting electrode. This method is considered to produce better images than the PE EIT system [29], [30]. However, no like-to-like comparison of the images from the two systems has yet been made.

In this study, a PE EIT system was developed to compare to the AE EIT system reported in [27]. The main difference between the two systems is that the AE EIT system uses 16 active ASICs mounted on a flexible PCB belt. Each electrode is assigned with its own V/I and IA, with a cable that connects it to the HUB. The PE EIT system has the same (single) ASIC on the HUB, and the excitation and recording are multiplexed through analog multipliers and via a cable to individual electrodes.

The electrode contact on the flexible PCB of the AE EIT system is soldered directly to the castellated hole on the resistive phantom shown in Fig. 6(b). As shown in Fig. 10 for the AE EIT system (b), in comparison with the PE EIT system (a), Global $F R=10.8$ and $R O I F R=1.3$. The Global $F R$ of the AE EIT system is slightly higher than the PE EIT system. This could be due to the mismatches between the 16 ASICs (e.g., uncalibrated IA gains).

To investigate this further, it is reported in [31] that in a clinical setting, other medical devices can emit electromagnetic signals that interfere with EIT recording. Such interference is observed when integrating the developed PE EIT system to a remote setting with a dedicated dc/dc supply. As shown in Fig. 9(a), interference is coupled to the recording signals when the cable of PE EIT system is closely placed over a medically 
approved power supply (TDK-Lambda, $75 \mathrm{~W}$ embedded switch mode power supply SMPS, $5 \mathrm{~V} \mathrm{dc}, \pm 12 \mathrm{~V} \mathrm{dc}$, open frame).

For comparison, the connection cable from active belt to the hub is placed over the same interference source for AE EIT system, as shown in Fig. 9(b). The impact of the supply interference on the EIT image is shown in Fig. 10(c) and (d). The coupled interference had a significant impact on the images that the PE EIT system produced; Global FR $=58.7$ and $R O I F R=16.9$. However, the performance of the AE EIT system was only slightly affected; Global $F R=17.8$ and $R O I F R=2.4$. This demonstrates that the AE system is very robust against electromagnetic interference.

\section{An Image Based FoM for EIT}

Using Global FR a new FoM for EIT system evaluation is proposed:

$$
\begin{aligned}
& { }^{F o M_{F R}}{ }_{P_{\text {system }}(\mathrm{W}) \times \text { Global } F R} \\
& f(\mathrm{~Hz}) \times \text { Frame Rate }(\text { frames } / \mathrm{s})
\end{aligned}
$$

where the variables are as previously defined in (7) and (9). It should be noted that from a clinical perspective, a higher frame rate offers higher temporal resolution [31]. For the PE system used in this paper, $P_{\text {system }}=1.38 \mathrm{~W}$, Global $F R=7.4, f=$ $125 \mathrm{kHz}$ and Frame Rate $=122$ frames $/ \mathrm{s}$, resulting in $F o M_{F R}=670 \frac{\mathrm{nW} \cdot \mathrm{s}}{\mathrm{Hz} \cdot \text { frames }}$.

\section{CONCLUSION}

Because common electrical parameters are not directly related to the quality of EIT images a simple and reproducible method to evaluate EIT systems has been proposed. It provides visually distinguishable hot colormap images and full reference objective image quality assessment. The method can be accessed through the GitHub repository [32]. Using the proposed Global FR and ROIFR metrics together with $F O M_{F R}$ which accounts for EIT operation bandwidth, image frame rate and system power consumption, evaluation and benchmarking of EIT systems is simpler and more straightforward. In addition, a PE EIT system has been evaluated and compared to a matched AE EIT system. For the first time, by evaluating the reconstructed image, this has shown the merit of AE EIT systems with respect to noise interference.

\section{REFERENCES}

[1] A. Romsauerova, A. McEwan, L. Horesh, R. Yerworth, R. H. Bayford, and D. S. Holder, "Multi-frequency electrical impedance tomography (EIT) of the adult human head: Initial findings in brain tumours, arteriovenous malformations and chronic stroke, development of an analysis method and calibration," Physiol. Meas., vol. 27, no. 5, pp. S147-161, 2006.

[2] J. Lee, S. Gweon, K. Lee, S. Um, K.-R. Lee, and H.-J. Yoo, "A 9.6$\mathrm{mW} / \mathrm{Ch} 10 \mathrm{-MHz}$ wide-bandwidth electrical impedance tomography IC with accurate phase compensation for early breast cancer detection," IEEE J. Solid-State Circuits, vol. 56, no. 3, pp. 887-898, 2021

[3] Y. Wu, D. Jiang, A. Bardill, S. De Gelidi, R. Bayford, and A. Demosthenous, "A high frame rate wearable EIT system using active electrode ASICs for lung respiration and heart rate monitoring," IEEE Trans. Circuits Syst. I Regul. Papers, vol. 65, no. 11, pp. 3810-3820, 2018.

[4] B. Liu et al., "A 13-channel 1.53-mW 11.28-mm2 electrical impedance tomography SoC based on frequency division multiplexing for lung physiological imaging," IEEE Trans. Biomed. Circuits Syst., vol. 13, no. 5, pp. 938-949, 2019.

[5] M. Yasin, S. Böhm, P. O. Gaggero, and A. Adler, "Evaluation of EIT system performance," Physiol. Meas., vol. 32, no. 7, pp. 851-865, 2011.

[6] M. Takhti and K. Odame, "Structured design methodology to achieve a high SNR electrical impedance tomography," IEEE Trans. Biomed. Circuits Syst., vol. 13, no. 2, pp. 364-375, 2019.

[7] A. McEwan, G. Cusick, and D. S. Holder, "A review of errors in multifrequency EIT instrumentation," Physiol. Meas., vol. 28, no. 7, pp. S197-S215, 2007.

[8] Z. Xu et al., "Development of a portable electrical impedance tomography system for biomedical applications," IEEE Sens. J., vol. 18, no. 18 , pp. 8117-8124, 2018.

[9] C. Tan, S. Liu, J. Jia, and F. Dong, "A wideband electrical impedance tomography system based on sensitive bioimpedance spectrum bandwidth," IEEE Trans. Instrum. Meas., vol. 69, no. 1, pp. 144-154, 2019.

[10] M. S. Tšoeu and M. R. Inggs, "Fully parallel electrical impedance tomography using code division multiplexing," IEEE Trans. Biomed. Circuits Syst., vol. 10, no. 3, pp. 556-566, 2016.

[11] L. Miao et al., "A new symmetric semi-parallel electrical impedance tomography (EIT) system - I: The design," in Proc. 2016 IEEE Int. Conf. Imaging Systems Techniq. (IST), Chania, Greece, 2016.

[12] EIDORS, "EIDORS: Electrical Impedance Tomography and Diffuse Optical Tomography Reconstruction Software." [Online]. Available: http://eidors3d.sourceforge.net/. [Accessed: 03-Oct-2019].

[13] A. Adler et al., "GREIT: a unified approach to 2D linear EIT reconstruction of lung images," Physiol. Meas., vol. 30, no. 6, pp. S35S55, 2009.

[14] Y. Wu, D. Jiang, N. Nazanin, F. H. Farnaz, and A. Demosthenous, "Towards a universal methodology for performance evaluation of electrical impedance tomography systems using full reference SNR," in Proc. 2020 IEEE Int. Symp. Circuits Syst. (ISCAS), Seville, Spain, 2020.

[15] K. Kim, J.-H. Kim, S. Gweon, M. Kim, and H.-J. Yoo, "A 0.5-V sub-10$\mu \mathrm{W} 15.28-\mathrm{m} \Omega / \sqrt{ } \mathrm{Hz}$ bio-impedance sensor IC with sub- $1^{\circ}$ phase error," IEEE J. Solid-State Circuits, vol. 55, no. 8, pp. 2161-2173, 2020.

[16] T. Zhang, H. Son, Y. Gao, J. Lan, and C.-H. Heng, "A 0.6V/0.9V 26.6to-1 $19.3 \mu \mathrm{W} \Delta \Sigma$-based bio-impedance readout IC with $101.9 \mathrm{~dB}$ SNR and $<0.1 \mathrm{~Hz}$ 1/f corner," in Proc. 2021 IEEE Int. Solid-State Circuits Conf. (ISSCC), San Francisco, CA, pp. 394-396, 2021.

[17] Y. Wu, F. F. Hanzaee, D. Jiang, R. H. Bayford, and A. Demosthenous, "Electrical impedance tomography for biomedical applications: Circuits and systems review," IEEE Open J. Circuits Syst., vol. 2, pp. 380-397, 2021.

[18] E. K. Murphy, M. Takhti, J. Skinner, R. J. Halter, and K. Odame, "Signal-to-noise ratio analysis of a phase-sensitive voltmeter for electrical impedance tomography," IEEE Trans. Biomed. Circuits Syst., vol. 11, no. 2, pp. 360-369, 2017.

[19] Y. Wu, D. Jiang, P. Langlois, R. Bayford, and A. Demosthenous, "A CMOS current driver with built-in common-mode signal reduction capability for EIT," in Proc. 43rd IEEE European Solid State Circuits Conf. (ESSCIRC), Leuven, Belgium, 2017, pp. 227-230.

[20] R. J. Yerworth, R. H. Bayford, G. Cusick, M. Conway, and D. S. Holder, "Design and performance of the UCLH Mark 1b 64 channel electrical impedance tomography (EIT) system, optimized for imaging brain function," Physiol. Meas., vol. 23, no. 1, pp. 149-158, 2002.

[21] A. Tizzard and R. H. Bayford, "Improving the finite element forward model of the human head by warping using elastic deformation," Physiol. Meas., vol. 28, no. 7, pp. S163--S182, 2007.

[22] M. Kim et al., "A $1.4 \mathrm{~m} \Omega$-sensitivity $94 \mathrm{~dB}$-dynamic-range electrical impedance tomography SoC and 48-channel hub SoC for 3D lung ventilation monitoring system," IEEE J. Solid-State Circuits, vol. 52, no. 11, pp. 2829-2842, 2017.

[23] M. Zamani, Y. Rezaeiyan, O. Shoaei, and W. A. Serdijn, “A $1.55 \mu \mathrm{W}$ bio-impedance measurement system for implantable cardiac pacemakers in $0.18 \mu \mathrm{m}$ CMOS," IEEE Trans. Biomed. Circuits Syst., vol. 12, no. 1, pp. 211-221, 2018.

[24] M. Takhti, Y. C. Teng, and K. Odame, "A $10 \mathrm{MHz}$ read-out chain for electrical impedance tomography," IEEE Trans. Biomed. Circuits Syst., vol. 12, no. 1, pp. 222-230, 2018.

[25] Y. Yang and J. Jia, "A multi-frequency electrical impedance tomography system for real-time 2D and 3D imaging," Rev. Sci. Instrum., vol. 88, no. 8, $085110,2017$.

[26] H. Gagnon, M. Cousineau, A. Adler, and A. E. Hartinger, "A resistive mesh phantom for assessing the performance of EIT systems," IEEE Trans. Biomed. Eng., vol. 57, no. 9, pp. 2257-2266, 2010. 
[27] Y. Wu, D. Jiang, A. Bardill, R. Bayford, and A. Demosthenous, "A 122 fps, $1 \mathrm{MHz}$ bandwidth multi-frequency wearable EIT belt featuring novel active electrode architecture for neonatal thorax vital sign monitoring," IEEE Trans. Biomed. Circuits Syst., vol. 13, no. 5, pp. $927-$ 937, 2019.

[28] S. Russo, S. Nefti-Meziani, N. Carbonaro, and A. Tognetti, "A quantitative evaluation of drive pattern selection for optimizing EITbased stretchable sensors," Sensors (Basel), vol. 17, 1999, 2017.

[29] B. Rigaud, Y. Shi, N. Chauveau, and J. P. Morucci, "Experimental acquisition system for impedance tomography with active electrode approach," Med. Biol. Eng. Comput., vol. 31, pp. 593-599, 1993.
[30] P. O. Gaggero, A. Adler, J. Brunner, and P. Seitz, "Electrical impedance tomography system based on active electrodes," Physiol. Meas., vol. 33, no. 5, pp. 831-847, 2012.

[31] I. Frerichs et al., "Chest electrical impedance tomography examination, data analysis, terminology, clinical use and recommendations: Consensus statement of the TRanslational EIT developmeNt stuDy group," Thorax, vol. 72, no. 1, pp. 83-93, 2017.

[32] EIT_FRFoM: An imaged based method for performance evaluation of EIT systems, 2021. [Online]. Available: https://github.com/BioZHIVE/EIT_FRFoM. 